\title{
PREFACE
}

\section{New Frontiers in Dysphagia Rehabilitation}

Swallowing problems affect as many as $40 \%$ of adults over age 60 years, and many millions of children also suffer from this devastating condition. Patients with dysphagia risk death from pneumonia, perhaps the most serious sequel of dysphagia, with malnutrition and dehydration additional dire secondary consequences. Some data are being ascertained on cost of dysphagia, for instance, in stroke patients, if they survive, indicating longer hospital stays and nursing home placements with diminished rehabilitation potential. Despite the devastating effect dysphagia has on health and on quality of life, evidence supporting the effects of specific interventions on swallowing outcomes is sparse.

The field of dysphagia treatment has evolved with predominantly two major categories of behavioral interventions: compensatory and rehabilitative approaches. The former, compensatory, which includes modification of the environment, such as providing a modified diet including thickened liquids, has developed primarily on the assumption that preservation of strength is critical to accomplishing the task of swallowing, and so, rather than depleting strength through rigorous oropharyngeal exercise, reserve to swallow and eat is optimized with the implementation of compensatory approaches by the patient or even by the caregiver.

For the past decade, environmental adaptation of diet modification has been a commonly recommended compensatory intervention for dysphagic patients, with management ranging from minimal restrictions to pureed diets or elimination of entire categories of liquids. Withholding thin liquids such as water, tea, or coffee and restricting fluid intake to thickened liquids is almost routine in hospitals and nursing homes in efforts to minimize or eliminate aspiration. Despite the huge effect that this unappealing practice may have on quality of life, these management strategies are frequently implemented in the absence of compelling published efficacy data.

Alternatively, active rehabilitation exercises, which are by nature more rigorous, with few exceptions, have been contraindicated because the activity is assumed to deplete any limited remaining swallowing functional reserve, thereby potentially exacerbating the dysphagia symptomatology. Sufficient treatment efficacy data are unavailable; thus, assumption-based patterns of practice have continued to prevail. Studies in recent years are confirming that swallowing is a function of the activation of large-scale distributed neural substrates involving various fiber systems from cortex to the periphery. Current assertions indicate that the swallow is not a reflex resistant to influence of behavioral modification, as has been believed for centuries. Thus, the will to swallow and control oral activity during the early phases of swallowing appear to offer access to what is more accurately referred to as the "patterned swallow response." Although disruption of this complex system centrally may contribute to impaired swallowing, restoration of swallowing function after injury, or even maintenance of swallowing in the presence of aging effects, also depends on neuromuscular morphologic factors such as muscle strength. To this end, optimal dysphagia rehabilitation relies on central neural plastic modifications as well as peripheral increase in muscle mass and strength, which may be accomplished by challenging both or either systems with emerging interventions, including exercise.

Our profession is only just beginning to provide evidence that, similar to other striated musculature, loss of bulbar innervated muscle strength and mass occurs with age. This

\footnotetext{
${ }^{1}$ Associate Director for Research GRECC, William S. Middleton Memorial Veterans Hospital; University of Wisconsin School of Medicine and Public Health, Madison, Wisconsin.

New Frontiers in Dysphagia Rehabilitation; Guest Editor, JoAnne Robbins, Ph.D., CCC-SLP, BRS-S.
}

Semin Speech Lang 2006;27:217-218. Copyright (C) 2006 by Thieme Medical Publishers, Inc., 333 Seventh Avenue, New York, NY 10001, USA. Tel: +1(212) 5844662.

DOI 10.1055/s-2006-955111. ISSN 0734-0478. 
condition, age-related muscle loss (sarcopenia), puts the older population at risk for dysphagia. Evidence is beginning to document that related loss of muscle strength with age is reversible with exercise. Speech language pathologists are recognizing the importance of implementing active exercise regimens in modes of delivery consistent with principles that promote neural plasticity. Neural plasticity is the mechanism by which the damaged brain "relearns" lost behavior in response to rehabilitation. Although all the factors that influence neural plasticity are yet to be defined or fully understood, certain key principles, such as repetition, intensity, and specificity, are emerging in the literature and are being increasingly incorporated into dysphagia practice.

In this issue of Seminars in Speech and Language, we assembled key clinicians and researchers who are translating neuroscience into clinical practice and refining clinical practice to elucidate how principles of neural plasticity can change swallowing function. Understanding and systematically manipulating the basic principles of neural plasticity that govern learning may permit identification of critical approaches that drive recovery.

The translation of the principles of neural plasticity to sensory stimulation or exercise and therapeutic approaches requires a paradigm shift in rehabilitation science. Current rehabilitation approaches typically do not implement principles in a standardized manner. The ability we have, as individual clinicians and as a group, to embrace these principles and integrate them into the mode of delivery of treatment is essential for evolving rehabilitation science in parallel with neuroscience.

Few dysphagic intervention methods have moved through the hierarchy of evidence that validates and establishes treatment procedures. Standardized protocols emerge in this volume-protocols that differ from traditional modes of treatment in speech pathology delivery in that these new protocols adhere to key principles of neural plasticity. Exercise principles that have existed for numerous decades are beginning to be used by a small group of speech and swallowing clinicians, researchers, and clinical researchers, some of whom are contributing authors in this volume, to underpin their efficacy-based studies of therapy. Exercise physiology principles and techniques that were based on clinical intuition in the past are now aligned with principles of neural plasticity and are beginning to be supported with objective measures for evidence-based designs.

Appealingly, the concept of providing sensory and exercise regimens to enhance neural plastic change tempts a reasonable speculation that stimulation of one sensorimotor system may result in positive neural plastic changes in the brain that relate to other sensorimotor systems. This phenomenon of transference may result in improvements of several functions as a result of therapy focused on a single target. For instance, swallowing may be improved as a result of exaggerated voice and speech exercise involving the respiratory, laryngeal, and articulatory mechanisms. Similarly speech articulation may improve as a result of strengthening the primary propulsive mechanism for swallowing; that is, the tongue and neighboring muscles and structures. There is sparse, but emerging, evidence that points to corollary influences of one sensorimotor system on another, and these corollary influences in a rehabilitative setting often are thought to augment the therapeutic results. However, there exists a logical possibility and emerging data that the opposite may be true in a system that is the functional agonist to the target system.

The articles in this issue of Seminars are unique. These "early" contributions describe a much-needed movement in dysphagia rehabilitation that combines old scientific principles with new and emerging technologies and design techniques. The authors have provided excellent and important information to move us all forward in the clinic, with the clear understanding that more information is essential to improve our patients and fulfill the potential provided by the discoveries emerging in this new frontier of dysphagia care. The systematic application of sensorimotor interventions according to the principles of neural plasticity selected a priori and incorporated into patient-oriented protocols defines a new frontier in dysphagia rehabilitation.

JoAnne Robbins, Ph.D., CCC-SLP, BRS-S

Guest Editor ${ }^{1}$ 